\title{
On Neumann problem for the degenerate Monge-Ampère type equations
}

Juhua Shi ${ }^{1}$ and Feida Jiang ${ }^{2^{*}}$ (B)

\section{${ }^{*}$ Correspondence:}

jfd2001@163.com

${ }^{2}$ School of Mathematics and

Shing-Tung Yau Center of Southeast

University, Southeast University,

211189 Nanjing, P.R. China

Full list of author information is

available at the end of the article

\begin{abstract}
In this paper, we study the global $C^{1,1}$ regularity for viscosity solution of the degenerate Monge-Ampère type equation $\operatorname{det}\left[D^{2} u-A(x, D u)\right]=B(x, u, D u)$ with the Neumann boundary value condition $D_{\nu} u=\varphi(x)$, where the matrix $A$ is under the regular condition and some structure conditions, and the right-hand term $B$ is nonnegative.
\end{abstract}

Keywords: Degenerate equations; Monge-Ampère type equations; Neumann problem; Global second order derivative estimates; Existence

\section{Introduction}

In this paper, we study the degenerate Monge-Ampère type equation

$$
\operatorname{det}\left[D^{2} u-A(\cdot, D u)\right]=B(\cdot, u, D u) \quad \text { in } \Omega,
$$

associated with the Neumann boundary value condition

$$
D_{\nu} u=\varphi(x) \quad \text { on } \partial \Omega \text {, }
$$

where $\Omega$ is a bounded domain, $D u$ and $D^{2} u$ denote the gradient and Hessian matrix of second order derivatives of the unknown function $u: \Omega \rightarrow \mathbb{R}$, respectively, $A: \Omega \times \mathbb{R}^{n} \rightarrow$ $\mathbb{R}^{n \times n}$ is a symmetric $n \times n$ matrix-valued function and $A \in C^{2}\left(\bar{\Omega} \times \mathbb{R}^{n}, \mathbb{R}^{n \times n}\right), B: \bar{\Omega} \times \mathbb{R} \times$ $\mathbb{R}^{n} \rightarrow \mathbb{R}^{+} \cup\{0\}$ is a nonnegative scalar function, $v$ denotes the unit inner normal on $\partial \Omega$ and $\varphi$ is a scalar-valued function defined on $\partial \Omega$. We shall use $x, z$, and $p$ to denote the points in $\Omega, \mathbb{R}$, and $\mathbb{R}^{n}$, respectively.

We introduce the definitions of nondegenerate and degenerate Monge-Ampère type equation respectively. The Monge-Ampère type equation (1) is called nondegenerate or degenerate if $D^{2} u-A$ is positive definite or nonnegative definite, namely the right-hand side term $B \geq B_{0}>0$ or $B \geq 0$, respectively, where $B_{0}$ is a constant. Accordingly, a solution $u$ of the Neumann boundary value problem (1)-(2) is called elliptic (degenerate elliptic) if $D^{2} u-A>0(\geq 0)$.

(c) The Author(s) 2021. This article is licensed under a Creative Commons Attribution 4.0 International License, which permits use, sharing, adaptation, distribution and reproduction in any medium or format, as long as you give appropriate credit to the original author(s) and the source, provide a link to the Creative Commons licence, and indicate if changes were made. The images or other third party material in this article are included in the article's Creative Commons licence, unless indicated otherwise in a credit line to the material. If material is not included in the article's Creative Commons licence and your intended use is not permitted by statutory regulation or exceeds the permitted use, you will need to obtain permission directly from the copyright holder. To view a copy of this licence, visit http://creativecommons.org/licenses/by/4.0/. 
We say that $A$ is strictly regular in $\Omega$, if

$$
\sum_{i, j, k, l=1}^{n} A_{i j}^{k l}(x, p) \xi_{i} \xi_{j} \eta_{k} \eta_{l} \geq c_{0}|\xi|^{2}|\eta|^{2}
$$

holds for all $(x, p) \in \Omega \times \mathbb{R}^{n}, \xi, \eta \in \mathbb{R}^{n}$ with $\xi \cdot \eta=0$, and some positive constant $c_{0}$, where $A_{i j}^{k l}=\frac{\partial^{2} A_{i j}}{\partial p_{k} \partial p_{l}}$. If $c_{0}$ on the right-hand side in (3) is replaced with 0 , we say that $A$ is regular in $\Omega$. As usual, the strictly regular condition and regular condition are also said to be the $\mathrm{A} 3$ condition and the A3w condition, respectively, see $[1,2]$.

For the Dirichlet boundary value problem, the regularity for degenerate MongeAmpère equation has been extensively studied, see [3-8]. When $A \equiv 0$, equation (1) reduces to the classical Monge-Ampère equation. The global $C^{1, \frac{1}{3}}$ regularity was obtained by Bao in [4] under the condition $B^{\frac{1}{n}}(x) \in C^{1,1}(\bar{\Omega})$. Guan, Trudinger, and Wang showed the global $C^{1,1}$ regularity in [7] with the relaxed condition $B^{\frac{1}{n-1}}(x) \in C^{1,1}(\bar{\Omega})$. Using the Pogorelov estimates independent of the lower bound $B$, the interior regularity was established by Błocki [5] and the authors [8]. When $A \not \equiv 0$, Andriyanova [3] proved the second order derivative estimates under the $\mathrm{A} 3 \mathrm{w}$ condition with the right-hand term $B(x, D u)=$ $q(x) \xi(x, D u)$, where $q^{\frac{1}{n-1}}(x) \in C^{1,1}(\bar{\Omega})$ is nonnegative and $\xi^{\frac{1}{n-1}}(x, D u) \in C^{1,1}\left(\bar{\Omega} \times \mathbb{R}^{n}\right)$ is positive.

When $A \neq 0$, the nondegenerate Monge-Ampère type equation (1) arises in various aspects such as optimal mass transportation problems, geometric optics, and conformal geometry (see, for instance, [1, 9-11]). Ma, Trudinger, and Wang [1] obtained a priori interior second order estimates under the A3 condition. The boundary $C^{2, \alpha}$ estimate for the Dirichlet problem was established by Huang et al. [12] under the A3 condition. Jiang et al. [13] obtained the global smooth solutions under the A3w condition. Furthermore, Leoper [2] proved that the $\mathrm{A} 3 \mathrm{w}$ condition is necessary for $C^{0}$ regularity of the optimal map in the optimal transportation problem.

For the Neumann boundary value problem, the global regularity of solutions for the classical Monge-Ampère equation was established by Lions, Trudinger, and Urbas in [14]. Subsequently, Jiang, Trudinger, and Xiang [15] proved the second order derivative estimates of solutions for the Monge-Ampère type equation (1) under the $\mathrm{A} 3 \mathrm{w}$ condition. However, as far as we know, there is no regularity result for the degenerate MongeAmpère type equation with Neumann boundary value condition, which is the motivation of this work.

Our global second derivative estimate is obtained by mixing the Bernstein type argument and the barrier argument. In order to construct the global barrier function under the regularity assumption of $A$, we need to assume the existence of a subsolution (strict subsolution) $\underline{u}$ to equation (1) satisfying

$$
\operatorname{det}\left[D^{2} \underline{u}-A(x, D \underline{u})\right] \geq(>) B(x, \underline{u}, D \underline{u}) \quad \text { in } \Omega(\text { on } \bar{\Omega})
$$

together with the Neumann boundary condition

$$
D_{\nu} \underline{u}=\varphi(x) \quad \text { on } \partial \Omega .
$$


In order to treat the degenerate case for equation (1), we assume that $B$ satisfies the following conditions:

$$
\begin{aligned}
& \left|B_{i}\right|,\left|B_{z}\right|,\left|B_{p_{i}}\right| \leq C_{1} B^{1-\frac{1}{2(n-1)}}, \\
& \left|B_{i j}\right|,\left|B_{i z}\right|,\left|B_{i p_{j}}\right|,\left|B_{z z}\right|,\left|B_{z p_{i}}\right|,\left|B_{p_{i} p_{j}}\right| \leq C_{2} B^{1-\frac{1}{n-1}}
\end{aligned}
$$

and

$$
\left\{B_{p_{i} p_{j}}-B_{p_{i}} B_{p_{j}}\right\}_{n \times n} \geq-C_{3} B^{2} I
$$

in $\Omega \times \mathbb{R} \times \mathbb{R}^{n}$ for $i, j=1, \ldots, n$, where $C_{1}, C_{2}$, and $C_{3}$ are positive constants, $I$ denotes the unit matrix, $B_{i}, B_{z}$, and $B_{p_{i}}$ denote the partial derivatives of $B$ with respect to $x_{i}, z$, and $p_{i}$, respectively. In fact, we can have the following relaxed version of condition (6) if $|B| \leq C$ :

$$
\left|B_{i}\right|,\left|B_{z}\right|,\left|B_{p_{i}}\right| \leq C_{4} B^{1-\frac{1}{n-1}}
$$

in $\Omega \times \mathbb{R} \times \mathbb{R}^{n}$ for $i=1, \ldots, n$, where $C_{4}$ depends on $n, \sup B$, and $C_{1}$.

We define the domain $\Omega$ to be uniformly $A$-convex with respect to $u \in C^{1}(\bar{\Omega})$ if

$$
\sum_{i, j=1}^{n}\left(D_{i} v_{j}(x)-A_{i j}^{k}(x, D u) v_{k}\right) \tau_{i} \tau_{j} \leq-\delta_{0} \quad \text { on } \partial \Omega
$$

for all vectors $\tau=\tau(x)$ tangent to $\partial \Omega$ and some positive constant $\delta_{0}$. For the Neumann problem, the definition of uniform $A$-convexity of $\Omega$ with respect to $u$ is introduced in [15]. We refer the readers to [15] for more descriptions of this definition. Based on (10), we can define a barrier function in the neighborhood of the boundary $\partial \Omega$ by using the defining function of the domain $\Omega$, which will be used in the double normal derivative estimate of $u$ on $\partial \Omega$ in Sect. 3.

We now formulate the main result.

Theorem 1.1 Let $\Omega$ be a bounded $C^{3,1}$ domain in $\mathbb{R}^{n}$ and $u \in C^{4}(\Omega) \cap C^{1,1}(\bar{\Omega})$ be an elliptic solution of Neumann (1)-(2). Assume that $\varphi \in C^{2,1}(\partial \Omega), A \in C^{2}\left(\bar{\Omega} \times \mathbb{R}^{n}, \mathbb{R}^{n \times n}\right)$ satisfies the $A 3$ w condition and $\Omega$ is uniformly $A$-convex with respect to $u$. Suppose that $B \in C^{1,1}(\bar{\Omega} \times$ $\left.\mathbb{R} \times \mathbb{R}^{n}\right)$ is a positive function satisfying conditions (6)-(8) and $B_{z}>0$. Assume that either of the following conditions holds:

(a) $A_{i j}=f_{i j}\left(x, u_{i}\right) \delta_{i j}$, where $f_{i j}(i, j=1, \ldots, n)$ are given functions and $\delta_{i j}$ is the usual Kronecker delta;

(b) There exists a sufficiently small constant $\delta$ depending on $n, A, B$, $\sup _{\Omega}|u|$ and $\sup _{\Omega}|D u|$ such that if $\left|D_{p_{k}} A_{i j}\right|<\delta$ for all $i, j, k=1, \ldots, n$.

Assume further that there exists a subsolution $\underline{u} \in C^{2}(\bar{\Omega})$ of Neumann problem (1)-(2) satisfying (4)-(5). Then we have the estimate

$$
\sup _{\Omega}\left|D^{2} u\right| \leq C
$$

where the constant $C$ depends on $n, \Omega,|A|_{2 ; \bar{\Omega}},|u|_{1 ; \bar{\Omega}}, \underline{u}, C_{1}, C_{2}, C_{3}, \sup _{\bar{\Omega}}|B|,\|\varphi\|_{C^{2,1}(\partial \Omega)}$, and $\delta_{0}$. 
We remark that the alternative conditions (a) and (b) for the matrix $A$ are used in the treatment of the third derivative terms in the proof, see the details in Sect. 3.

Since the global second order derivative estimates are independent of the lower bound of $B$, we have the following existence result by approximation. Before stating the theorem, we introduce the definition of viscosity solution of equation (1). An upper (lower)semicontinuous function $u$ is called a viscosity subsolution (supersolution) of equation (1) if, for any function $\phi \in C^{2}(\Omega)$ such that $u-\phi$ has a local maximum (minimum) at some point $x_{0} \in \Omega$, there holds

$$
\operatorname{det}\left\{D^{2} \phi\left(x_{0}\right)-\left[A\left(x_{0}, D \phi\left(x_{0}\right)\right)\right]\right\} \geq(\leq) B\left(x_{0}, u\left(x_{0}\right), D \phi\left(x_{0}\right)\right) .
$$

A continuous function $u$ is a viscosity solution of equation (1) if it is both a viscosity subsolution and a viscosity supersolution of equation (1).

Based on the a priori second derivative estimate, we now establish the existence result in the following theorem.

Theorem 1.2 Under the assumption of Theorem 1.1, assume that " $B$ is a nonnegative function" and "a strict subsolution $\underline{u} \in C^{2}(\bar{\Omega})$ " in place of "B is a positive function" and "a subsolution $\underline{u} \in C^{2}(\bar{\Omega})$ ", respectively. Assume further that there exists a supersolution $\bar{u}$ of equation (1) satisfying $D_{\nu} \bar{u} \leq \varphi(x)$ on $\partial \Omega$, and A satisfies the quadratic structure condition from below, namely

$$
A(x, p) \geq-\mu_{0}\left(1+|p|^{2}\right) I
$$

where $\mu_{0}$ is a positive constant depending on sup $|u|$ and I denotes the $n \times n$ identity matrix. Then there exists a degenerate elliptic viscosity solution $u \in C^{1,1}(\bar{\Omega})$ for the Neumann problem (1)-(2).

Remark 1 Note that in Theorem 1.2, if $B$ is a positive function and $\underline{u} \in C^{2}(\bar{\Omega})$ is merely a subsolution, we can get the existence and uniqueness of the elliptic solution $u \in C^{3, \alpha}(\bar{\Omega})$ of problem (1)-(2) for some $\alpha \in(0,1)$. We can prove this by following the proof of Theorem 1.2 in [15] and replacing $\bar{u}$ in its original proof with $\underline{u}$. The reason why we can replace $\bar{u}$ with $\underline{u}$ is that both $A$ in (1) and $\varphi$ in (2) have no $u$ dependence. Indeed, when $A$ in (1) is independent of $u$, we can obtain Lemma 2.2, which can replace Lemma 2.1 of [15]. Meanwhile, since $\varphi$ in (2) has no $u$ dependence, we are still able to get the key inequality (47) in our proof. Then we can follow the proof of Theorem 1.2 in [15] to establish $u \in C^{3, \alpha}(\bar{\Omega})$.

Remark 2 It is clear that Theorem 1.2 can be applied to the Monge-Ampère equation with homogenous right-hand side as follows:

$$
\operatorname{det}\left[D^{2} u-A(x, D u)\right]=c_{0}|x|^{\alpha} \quad \text { in } \Omega,
$$

where $c_{0}$ is a nonnegative constant, $\alpha \in[2(n-1),+\infty)$ is a constant. Thus, there exists a unique convex solution $u \in C^{1,1}(\bar{\Omega})$ of equation (14) under the Neumann boundary value condition (2). For the Dirichlet problem of the totally degenerate Monge-Ampère equation (14) when $c_{0}=0$ and $A \equiv 0$, we refer the reader to [16-18]. 
Remark 3 Condition (a) in Theorems 1.1 and 1.2 can include $A=\operatorname{diag}\left(u_{1}^{2}, \ldots, u_{n}^{2}\right)$ as an example, which satisfies both the A3w condition and the structure condition (13). Both conditions (a) and (b) in Theorems 1.1 and 1.2 can cover the special case when the matrix $A$ is independent of $p$, so that the standard Monge-Ampère case is already involved. It would be interesting to relax restrictions (a) or (b) of the matrix $A$ in Theorems 1.1 and 1.2.

The paper is organized as follows. In Sect. 2, we introduce two lemmas which will be used to deal with the degenerate term and in the construction of the global barrier function, respectively. In Sect. 3, we give the proof of Theorem 1.1 which constitutes the core of this paper. In this section, we reduce the global estimates to the boundary by using a global auxiliary function, where the computations of the third order derivatives are carried out in detail under the two alternative conditions of Theorem 1.1. For the boundary estimates, we construct a suitable barrier function which can be used to control the degenerate term, originating from the differentiation of equation (1). In this part, we mix the techniques of dealing with the Neumann problems for nondegenerate Monge-Ampère type equations in $[14,15]$ and the Dirichlet problems for degenerate Monge-Ampère type equations in [3, 5-7]. In Sect. 4, we complete the proof of Theorem 1.2.

\section{Preliminaries}

In this section, we introduce two lemmas. One lemma is used to deal with the degenerate term which arises in differentiating equation (1). The other lemma is used to construct the global barrier function. In equation (1), we first suppose $B>0$ in $\Omega$,

$$
\left\{\tilde{u}_{i j}\right\}:=\left\{u_{i j}-A_{i j}\right\} \quad \text { and } \quad\left\{\tilde{u}^{i j}\right\}:=\left\{\tilde{u}_{i j}\right\}^{-1} \text {. }
$$

Then both the matrices $\left\{\tilde{u}_{i j}\right\}$ and $\left\{\tilde{u}^{i j}\right\}$ are positive definite. We can rewrite equation (1) in the form

$$
\log \left[\operatorname{det}\left(\tilde{u}_{i j}\right)\right]=\tilde{B} \quad \text { in } \Omega,
$$

where $\tilde{B}:=\log B$. By differentiating equation (15) in the direction $\xi \in \mathbb{R}^{n}$ once and twice respectively, we have

$$
\tilde{u}^{i j}\left(D_{\xi} u_{i j}-A_{i j, k} \xi_{k}-A_{i j}^{k} D_{\xi} u_{k}\right)=D_{\xi} \tilde{B}
$$

and

$$
\begin{aligned}
& \tilde{u}^{i j}\left(D_{\xi \xi} u_{i j}-A_{i j, k l} \xi_{k} \xi_{l}-A_{i j}^{k l} D_{\xi} u_{k} D_{\xi} u_{l}-A_{i j}^{k} D_{\xi \xi} u_{k}-2 A_{i j, l}^{k} \xi_{l} D_{\xi} u_{k}\right) \\
& \quad=\tilde{u}^{i s} \tilde{u}^{j t} D_{\xi} \tilde{u}_{i j} D_{\xi} \tilde{u}_{s t}+D_{\xi \xi} \tilde{B},
\end{aligned}
$$

where

$$
D_{\xi} \tilde{B}=\frac{B_{\xi}+B_{z} D_{\xi} u+B_{p_{k}} D_{k \xi} u}{B}
$$


and

$$
\begin{aligned}
D_{\xi \xi} \tilde{B}= & \frac{B_{\xi \xi}+B_{z z}\left(D_{\xi} u\right)^{2}+B_{z} D_{\xi \xi} u+B_{p_{k}} D_{\xi \xi} u_{k}+B_{p_{k} p_{l}}\left(D_{\xi} u_{l}\right)\left(D_{\xi} u_{k}\right)}{B} \\
& +\frac{2 B_{\xi z} D_{\xi} u+2 B_{\xi p_{l}} D_{\xi} u_{l}+2 B_{z p_{k}}\left(D_{\xi} u\right)\left(D_{\xi} u_{k}\right)}{B} \\
& -\frac{B_{\xi}^{2}+B_{z}^{2}\left(D_{\xi} u\right)^{2}+B_{p_{k}} B_{p_{l}}\left(D_{\xi} u_{k}\right)\left(D_{\xi} u_{l}\right)}{B^{2}} \\
& -\frac{2 B_{\xi} B_{z} u_{\xi}+2 B_{\xi} B_{p_{l}} D_{\xi} u_{l}+2 B_{z} B_{p_{l}}\left(D_{\xi} u\right)\left(D_{\xi} u_{l}\right)}{B^{2}},
\end{aligned}
$$

where $B_{i}=\frac{\partial B}{\partial x_{i}}, B_{z}=\frac{\partial B}{\partial z}, B_{p_{k}}=\frac{\partial B}{\partial p_{k}}, A_{i j, k}=\frac{\partial A_{i j}}{\partial x_{k}}, A_{i j}^{k}=\frac{\partial A_{i j}}{\partial p_{k}}$,

$$
D_{i} v=\frac{\partial v}{\partial x_{i}}+\frac{\partial v}{\partial z} D_{i} u+\frac{\partial v}{\partial p_{k}} D_{k i} u=v_{i}+v_{z} D_{i} u+v_{p_{k}} D_{k i} u
$$

and

$$
D_{\xi} v=D_{i} v \xi_{i}=v_{i} \xi_{i}+v_{z} D_{i} u \xi_{i}+v_{p_{k}} D_{k i} u \xi_{i}
$$

for a function $v=v(x, z, p)$. Note that we use the standard summation convention in the context that repeated indices indicate summation from 1 to $n$ unless otherwise specified.

Next, we point out several facts that will be used in the proof of Theorem 1.1.

Lemma 2.1 Let $u \in C^{3}(\Omega)$ be a solution of equation (1) in a bounded domain $\Omega \subset \mathbb{R}^{n}$. Assume that $B$ satisfies (6), $B>0$, and $\tilde{B}=\log B$. Then we have the following properties:

(i)

$$
\left|D_{i} \tilde{B}\right| \leq C\left[1+\max _{j}\left(\left|\tilde{u}_{i j}\right|\right)\right] B^{-\frac{1}{n-1}}
$$

holds for $i=1, \ldots, n$, where the constant $C$ depends on $n, C_{1}, A$, and $\|u\|_{C^{1}(\bar{\Omega})}$.

(ii) If $B$ also satisfies conditions (7)-(8), then

$$
D_{i i} \tilde{B} \geq-C\left[1+\max _{j}\left(\left|\tilde{u}_{i j}\right|\right)\right] B^{-\frac{1}{n-1}}-C^{\prime}\left[1+\max _{j}\left(\left|\tilde{u}_{i j}\right|\right)\right]^{2}+\sum_{k=1}^{n} \tilde{B}_{p_{k}} D_{i i} u_{k}
$$

holds for $i=1, \ldots, n$, where the constant $C$ depends on $n, C_{1}, C_{2}, A$, and $\|u\|_{C^{1}(\bar{\Omega})}$, and the constant $C^{\prime}$ depends on $C_{3}$ and $A$.

Lemma 2.1 can be proved by direct calculations using (6) (or (9)), (7), and (8). We briefly prove it here. A more detailed proof can be found in [19].

Proof of Lemma 2.1 By choosing $\xi=e_{i}$ in (18) and using $\tilde{u}_{i j}=u_{i j}-A_{i j}$ and (9) (the relaxed version of (6)), we get (20) and finish the proof of conclusion (i).

Next, we turn to proving (ii). By condition (8), we have

$$
\begin{aligned}
\frac{B_{p_{k} p_{l}} B-B_{p_{k}} B_{p_{l}}}{B^{2}} u_{i l} u_{i k} & \geq-C_{3} \delta_{k l}\left(\tilde{u}_{i l}+A_{i l}\right)\left(\tilde{u}_{i k}+A_{i k}\right) \\
& \geq-C^{\prime}\left[1+\max _{j}\left(\left|\tilde{u}_{i j}\right|\right)\right]^{2}
\end{aligned}
$$


where $\delta_{k l}$ denotes the usual Kronecker delta, the constant $C^{\prime}$ depends on $C_{3}$ and $A$. It follows from (6) and (7) that the sum of the other terms on the right-hand side of (7) has a lower bound $-C\left[1+\max _{j}\left(\left|\tilde{u}_{i j}\right|\right)\right] B^{-\frac{1}{n-1}}$, where the constant $C$ depends on $n, C_{1}, C_{2}$, sup $|B|$, $A$, and $\sup _{\Omega}|D u|$.

Using the lower bounds $-C\left[1+\max _{j}\left(\left|\tilde{u}_{i j}\right|\right)\right] B^{-\frac{1}{n-1}}$ and (22), and taking $\xi=e_{1}$ in (19), we get (21) and finish the proof of conclusion (ii).

Remark 4. We remark that $\tilde{B}=\log B$ satisfies condition (8) if it is semi-convex in $p$. The term $\sum_{k=1}^{n} \tilde{B}_{p_{k}} D_{i i} u_{k}$ on the right-hand side of (21) can also be dealt with directly. We shall explain the treatment in the later discussion.

We introduce the following linearized operator of equation (1):

$$
L=\sum_{i, j, k=1}^{n}\left[\tilde{u}^{i j}\left(D_{i j}-A_{i j}^{k}(x, D u) D_{k}\right)-\tilde{B}_{p_{k}} D_{k}\right] .
$$

We introduce a fundamental lemma, which is important for constructing the global barrier function in the next section.

Lemma 2.2 Let $u \in C^{2}(\bar{\Omega})$ be an elliptic solution of (1), and let $\underline{u} \in C^{2}(\bar{\Omega})$ be an elliptic function of equation (1) satisfying $D^{2} \underline{u}-A(x, D \underline{u}) \geq \delta I>0$ in $\bar{\Omega}$ for some positive constant $\delta$, $A$ is regular and $B \in C^{1,1}\left(\bar{\Omega} \times \mathbb{R} \times \mathbb{R}^{n}\right)$ is a positive function satisfying (6). Then

$$
L\left(e^{K(\underline{u}-u)}\right) \geq \epsilon_{1} \mathcal{T}-C\left(B^{-\frac{1}{2(n-1)}}+1\right)
$$

holds in $\Omega$ for sufficiently large positive constant $K$ and uniform positive constants $\epsilon_{1}, C$ depending on $\|A\|_{C^{2}},\|B\|_{C^{1,1}}, \Omega,\|u\|_{C^{1}},\|\underline{u}\|_{C^{1}}, \delta$, and $C_{1}$ in (6), where $L$ is the operator in (23), and $\mathcal{T}=\sum_{i}^{n} \tilde{u}^{i i}$.

Proof Since $A$ is regular, similar to Lemma 2.1(ii) in [20], we have

$$
\begin{aligned}
L\left(e^{K(\underline{u}-u)}\right) \geq & \epsilon_{1}(\mathcal{T}+1)+C\left\{\log \frac{\operatorname{det}\left[D^{2} \underline{u}-A(x, D \underline{u})\right]}{\operatorname{det}\left[D^{2} u-A(x, D u)\right]}\right. \\
& \left.-\sum_{k=1}^{n} \tilde{B}_{p_{k}}(x, u, D u) D_{k}(\underline{u}-u)\right\} \\
\geq & \epsilon_{1} \mathcal{T}+\epsilon_{1}+C\left[\log \frac{\delta^{n}}{M_{1}}-B^{-\frac{1}{2(n-1)}}\right],
\end{aligned}
$$

where $D^{2} \underline{u}-A(x, D \underline{u}) \geq \delta I>0$ and condition (6) are used in the second inequality of (25), $M_{1}=\sup _{x \in \Omega} B(x, u, D u)$, and the constant $C$ changes from line to line. Then estimate (24) follows by further adjusting the constant $C$.

Remark 5 Note that the barrier inequality in Lemma 2.2 is different from that in [15]. Here the constants $\epsilon_{1}$ and $C$ in estimate (24) of Lemma 2.2 are independent of the positive lower bound of $B$. Note also that estimate (24) is proved under condition (6), while in [19] a similar estimate is proved under condition (8). 


\section{Global second order derivative estimates}

In this section, by constructing a global auxiliary function, we can reduce the global estimates to the boundary. On the boundary, in order to get the mixed tangential normal second order derivatives of $u$, we apply the tangential operator to the boundary value condition (2). A bound of double normal derivative of $u$ on $\partial \Omega$ is obtained by constructing a suitable auxiliary function. We use the key trick of [14] to establish the global second order derivative estimates of solutions for the degenerate Monge-Ampère type equations with the Neumann boundary value condition. For the argument below, we assume that the functions $\varphi, v$ are smoothly extended to $\bar{\Omega}$. The constant $C$ in this section changes from line to line.

Mixed tangential normal derivative estimate on $\partial \Omega$. We introduce the tangential gradient operator $\delta=\left(\delta_{1}, \delta_{2}, \ldots, \delta_{n}\right)$, where $\delta_{i}=\sum_{j=1}^{n}\left(\delta_{i j}-v_{i} v_{j}\right) D_{j}$ for $i=1, \ldots, n$. Applying this tangential operator to boundary condition (2), we have

$$
\left(D_{k} u\right)\left(\delta_{i} v_{k}\right)+v_{k}\left(\delta_{i} D_{k} u\right)=\delta_{i} \varphi \quad \text { on } \partial \Omega
$$

If $\tau$ is a direction tangential to $\partial \Omega$ at any point $y \in \partial \Omega$, we have

$$
\begin{aligned}
D_{\tau \nu} u(y) & =\sum_{i, k=1}^{n} \tau_{i} v_{k} D_{i k} u=\sum_{i, k=1}^{n} \tau_{i} v_{k} D_{i k} u-\sum_{i, j, k=1}^{n} \tau_{i} v_{k} v_{i} v_{j} D_{j k} u \\
& =\sum_{i, j, k=1}^{n} \tau_{i} v_{k}\left(\delta_{i j}-v_{i} v_{j}\right) D_{j k} u=\sum_{i, k=1}^{n} \tau_{i} v_{k} \delta_{i} D_{k} u \\
& =\sum_{i, k=1} \tau_{i} \delta_{i} \varphi-\tau_{i}\left(\delta_{i} v_{k}\right) D_{k} u=\sum_{i, k=1} \tau_{i} D_{i} \varphi-\tau_{i}\left(D_{i} v_{k}\right) D_{k} u
\end{aligned}
$$

where the second equality and the last equality are both valid using the fact that $\tau \cdot v=0$, and the fifth equality holds by (26). Hence, from (27) we obtain

$$
\left|D_{\tau \nu} u\right| \leq C
$$

on $\partial \Omega$, where the constant $C$ depends on $\sup _{\bar{\Omega}}|D u|$ and $\Omega$.

Double normal derivative estimate on $\partial \Omega$. We may consider any boundary point. Without loss of generality, we may take it to be the origin and the $x_{n}$-axis in the direction of the interior normal. From the uniform $A$-convexity of $\Omega$ and the regularity of $A$, there exists a $C^{2}$ defining function in $\bar{\Omega}$ satisfying

$$
\phi=0 \quad \text { on } \partial \Omega, \quad D_{\nu} \phi=-1 \quad \text { on } \partial \Omega, \quad \text { and } \quad \phi<0 \quad \text { in } \Omega \text {, }
$$

together with the inequality

$$
\left\{D_{i j} \phi-A_{i j}^{k}(x, D u) D_{k} \phi\right\}_{n \times n} \geq \delta_{0} I,
$$

in a neighborhood $\mathcal{N}$ of $\partial \Omega$, whenever $D_{v} u \geq \varphi(x)$, where $\delta_{0}$ is a positive constant and $I$ denotes the identity matrix. We employ the auxiliary function

$$
w= \pm\left(D_{\nu} u-\varphi(x)\right)+\beta \phi
$$


in

$$
\Omega_{d_{0}}:=\left\{x \in \Omega \mid \operatorname{dist}(x, \partial \Omega)<d_{0}\right\},
$$

where $\beta$ and $d_{0}$ are two positive constants to be determined. By the continuity of $D_{\nu} \phi$ and $D_{\nu} \phi=-1$ on $\partial \Omega$, there exists a small constant $d_{0}$ such that $D_{\nu} \phi \leq-\frac{1}{2}$ in $\Omega_{d_{0}}$.

It follows from (16) that

$$
\begin{aligned}
L w= & \pm \tilde{u}^{i j}\left[D_{i j}\left(v_{k} D_{k} u\right)-A_{i j}^{l} D_{l}\left(v_{k} D_{k} u\right)\right] \mp L \varphi+\beta L \phi \\
= & \pm \tilde{u}^{i j}\left[\left(D_{i j} v_{k}\right) D_{k} u+2 D_{j} v_{k} D_{i k} u-A_{i j}^{l}\left(D_{l} v_{k}\right) D_{k} u+v_{k} A_{i j, k}\right] \\
& +v_{k} \tilde{B}_{p_{k}} \mp L \varphi+\beta L \phi \\
\geq & \beta L \phi-C \mathcal{T}-C-C B^{-\frac{1}{n-1}} \\
\geq & \beta L \phi-C \mathcal{T}-C B^{-\frac{1}{n-1}}
\end{aligned}
$$

where $\mathcal{T}=\sum_{i=1}^{n} \tilde{u}^{i i}$, the first inequality is obtained by using (20) in Lemma 2.1 , the second inequality is obtained by using the fact that

$$
\frac{1}{n} \mathcal{T} \geq\left(\prod_{i=1}^{n} \tilde{u}^{i i}\right)^{\frac{1}{n}}=\left(\prod_{i=1}^{n} \tilde{u}_{i i}\right)^{-\frac{1}{n}}=B^{-\frac{1}{n}} \geq C .
$$

By a direct calculation, we obtain

$$
\begin{aligned}
L \phi & =\tilde{u}^{i j}\left[D_{i j} \phi-A_{i j}^{k}(x, D u) D_{k} \phi\right]-\tilde{B}_{p_{k}} D_{k} \phi \\
& \geq \delta_{0} \mathcal{T}-\tilde{B}_{p_{k}} D_{k} \phi \\
& \geq \delta_{0} \mathcal{T}-C B^{-\frac{1}{2(n-1)}}
\end{aligned}
$$

where the first and second inequalities are established by using (34) and (6), respectively. Therefore, from (32), (33), and (34), we have

$$
L w \geq\left(\beta \delta_{0}-C\right) \mathcal{T}-C B^{-\frac{1}{n-1}}-C \beta B^{-\frac{1}{2(n-1)}}
$$

We decompose $\Omega_{d_{0}}=\Omega_{d_{0}}^{1} \cup \Omega_{d_{0}}^{2}$, where

$$
\Omega_{d_{0}}^{1}=\Omega_{d_{0}} \cap\left\{\sum_{i=1}^{n} \tilde{u}_{i i} \geq n\right\}
$$

and

$$
\Omega_{d_{0}}^{2}=\Omega_{d_{0}} \cap\left\{\sum_{i=1}^{n} \tilde{u}_{i i}<n\right\} .
$$

If $\max _{\bar{\Omega}_{d_{0}}} w=w(\bar{x})$ for some $\bar{x} \in \bar{\Omega}_{d_{0}}$. The proof of the double normal derivative estimate of $u$ on $\partial \Omega$ splits into three stages according to whether $\bar{x} \in \Omega_{d_{0}}^{1}$ or $\bar{x} \in \Omega_{d_{0}}^{2}$, or neither. 
Case 1. $\bar{x} \in \Omega_{d_{0}}^{1}$. Since $\max _{\bar{\Omega}_{d_{0}}} w=w(\bar{x})$ for $\bar{x} \in \Omega_{d_{0}}^{1}$, then we have

$$
L w(\bar{x}) \leq 0
$$

We may assume that $\tilde{u}_{i j}(\bar{x})$ is diagonal. Without loss of generality, we may assume that $\tilde{u}_{11}(\bar{x}) \geq 1$. At the point $\bar{x}$, we have

$$
\begin{aligned}
\mathcal{T} & \geq \sum_{i=2}^{n} \tilde{u}^{i i} \\
& \geq(n-1)\left(\prod_{i=2}^{n} \tilde{u}^{i i}\right)^{\frac{1}{n-1}} \\
& =(n-1)\left(\prod_{i=1}^{n} \tilde{u}^{i i}\right)^{\frac{1}{n-1}}\left(\tilde{u}_{11}\right)^{\frac{1}{n-1}} \\
& \geq(n-1) B^{-\frac{1}{n-1}},
\end{aligned}
$$

where $\tilde{u}_{11}(\bar{x}) \geq 1$ is used in the last inequality. At $\bar{x}$, we can assume that $\sqrt{\mathcal{T}} \geq \frac{3 C}{\delta_{0} \sqrt{n-1}}$, otherwise we have already got the global second order derivative estimates. Therefore at $\bar{x}$, by (35), we have

$$
\begin{aligned}
L w & \geq\left(\frac{\beta \delta_{0}}{3}-C\right) \mathcal{T}+\frac{\beta \delta_{0}}{3} \mathcal{T}-C B^{-\frac{1}{n-1}}+\frac{\beta \delta_{0}}{3} \mathcal{T}-C \beta B^{-\frac{1}{2(n-1)}} \\
& \geq\left(\frac{\beta \delta_{0}}{3}-C\right) \mathcal{T}+\left(\frac{\beta \delta_{0}}{3}-\frac{C}{n-1}\right) \mathcal{T}+\left(\frac{\beta \delta_{0}}{3} \sqrt{\mathcal{T}}-\frac{C \beta}{\sqrt{n-1}}\right) \sqrt{\mathcal{T}} \\
& >0
\end{aligned}
$$

where we choose $\beta \geq \frac{3 C}{\delta_{0}}+1$ such that the last inequality holds. It is a contradiction with (36). Therefore, $\bar{x} \notin \Omega_{d_{0}}^{1}$.

Case 2. $\bar{x} \in \Omega_{d_{0}}^{2}$. For the unit inner normal vector $v=\left(v_{1}, \ldots, v_{n}\right)$, we must have

$$
0 \leq\left|v_{i}\right| \leq 1 \quad \text { for } i=1, \ldots, n \text {. }
$$

Since $\max _{\bar{\Omega}_{d_{0}}^{2}} w=w(\bar{x}), \bar{x}=\left(\bar{x}_{1}, \ldots, \bar{x}_{n}\right) \in \Omega_{d_{0}}^{2}$, we obtain

$$
w_{v}(\bar{x})=0
$$

Therefore, we have at $\bar{x}$

$$
\begin{aligned}
0 & =w_{\nu}= \pm\left(D_{\nu \nu} u-D_{\nu} \varphi\right)+\beta D_{\nu} \phi \\
& = \pm\left(\sum_{i, j}^{n} v_{i} v_{j} D_{i j} u\right) \mp D_{\nu} \varphi+\beta D_{\nu} \phi \\
& \leq n^{2} \sum_{i=1}^{n} \tilde{u}_{i i} \mp D_{\nu} \varphi+\beta D_{\nu} \phi .
\end{aligned}
$$


Choose $\beta \geq 2 \max |D \varphi|+4 n^{3}$ such that $\sum_{i=1}^{n} \tilde{u}_{i i} \geq 2 n$, which is a contradiction with $\sum_{i=1}^{n} \tilde{u}_{i i}<n$. Therefore, $\bar{x} \notin \Omega_{d_{0}}^{2}$.

Case 3. $\bar{x} \in \partial \Omega_{d_{0}}$. On $\partial \Omega_{d_{0}} \cup \Omega$, we have

$$
w= \pm\left(D_{v} u-\varphi\right)+\beta \phi \leq 0
$$

where $\beta$ is chosen large enough such that the last inequality holds. On $\partial \Omega$, we have

$$
w= \pm\left(D_{v} u-\varphi(x)\right)+\beta \phi=0
$$

Therefore, for all $x \in \bar{\Omega}_{d_{0}}$, we have

$$
w(x) \leq 0 .
$$

Since $w(0)=0$, we have

$$
D_{\nu} w(0) \leq 0,
$$

which implies

$$
\left|D_{v v} u\right| \leq C \quad \text { on } \partial \Omega
$$

Remark 6 In the above proof, we only use (30) in a neighborhood $\mathcal{N}$ of $\partial \Omega$ whenever $D_{v} u \geq \varphi(x)$. If $D_{v} u<\varphi(x)$ in a neighborhood $\mathcal{N}_{0}$ of some boundary point $x_{0} \in \partial \Omega$, from the boundary condition, we directly get an upper bound $D_{v v} u\left(x_{0}\right) \leq D_{\nu} \varphi\left(x_{0}\right)$. While the lower bound $D_{v v} u \geq \sum_{i, j}^{n} A_{i j} v_{i} v_{j}$ at $x_{0}$ can be derived from the ellipticity.

Based on the mixed tangential normal derivative estimate and the double normal derivative estimate on $\partial \Omega$, we now use the method in [15] to prove Theorem 1.1, which is a modification of the original method in [14].

Proof of Theorem 1.1 We modify the elliptic subsolution $\underline{u}$ by adding a perturbation function $a \phi$, where $a$ is a small positive constant and $\phi$ is the defining function of the domain $\Omega$ satisfying (29). Note that the function $\underset{\sim}{u}:=\underline{u}-a \phi$ is still uniformly elliptic in $\Omega$ if $a$ is a sufficiently small. By a direct computation, we have

$$
\begin{aligned}
D_{\nu}(\underline{u}-u) & =D_{\nu} \underline{u}-D_{\nu} u-a D_{\nu} \phi \\
& =-a D_{\nu} \phi \\
& =a
\end{aligned}
$$

on $\partial \Omega$. We define a function with the form $\Phi=\exp [K(\underset{\sim}{u}-u)]$ with a positive constant $K$. Replacing $\underline{u}$ in Lemma 2.2 with $\underset{\sim}{u}$, from (24), we have

$$
L \Phi \geq \epsilon_{1} \mathcal{T}-C\left(B^{-\frac{1}{2(n-1)}}+1\right) \quad \text { in } \Omega
$$


By a direct calculation, we also have

$$
D_{\nu} \Phi \geq K a \inf \left(e^{K(\underline{u}-u)}\right)>0 \quad \text { on } \partial \Omega
$$

We employ the auxiliary function

$$
G(x, \xi)=\left(\tilde{u}_{\xi \xi}-v^{\prime}(x, \xi)\right) e^{\frac{\alpha}{2}|D u|^{2}+\kappa \Phi}
$$

in $\bar{\Omega} \times \mathbb{R}^{n}$, where $|\xi|=1, \alpha$ and $\kappa$ are positive constants to be determined, $v^{\prime}$ is given by

$$
v^{\prime}(x, \xi)=2(\xi \cdot v) \xi_{i}^{\prime}\left(D_{i} \varphi(x)-D_{k} u D_{i} v_{k}-A_{i j} v_{j}\right),
$$

and $\xi^{\prime}$ is given by

$$
\xi^{\prime}=\xi-(\xi \cdot v) \nu
$$

Assume that $G$ attains its maximum at $x_{0} \in \Omega$ and $\xi=\xi_{0}$. Let

$$
H(x, \xi)=\log G(x, \xi)=\log \left(\tilde{u}_{\xi \xi}-v^{\prime}\right)+\frac{\alpha}{2}|D u|^{2}+\kappa \Phi
$$

then the function $H$ attains its maximum at $x_{0} \in \Omega$ and $\xi=\xi_{0}$. From now on, all the calculations will be made at the point $x=x_{0}$ and $\xi=\xi_{0}$ unless otherwise specified. At $x_{0}$, we obtain

$$
\begin{aligned}
0= & D_{i} H=\frac{D_{i}\left(\tilde{u}_{\xi \xi}-v^{\prime}\right)}{\tilde{u}_{\xi \xi}-v^{\prime}}+\alpha D_{k} u D_{i k} u+\kappa D_{i} \Phi, \text { for } i=1, \ldots, n, \\
0 \geq & D_{i j} H \\
= & \frac{D_{i j}\left(\tilde{u}_{\xi \xi}-v^{\prime}\right)}{\tilde{u}_{\xi \xi}-v^{\prime}}-\frac{D_{i}\left(\tilde{u}_{\xi \xi}-v^{\prime}\right) D_{j}\left(\tilde{u}_{\xi \xi}-v^{\prime}\right)}{\left(\tilde{u}_{\xi \xi}-v^{\prime}\right)^{2}} \\
& +\alpha\left(D_{i k} u D_{j k} u+D_{k} u D_{i j k} u\right)+\kappa D_{i j} \Phi .
\end{aligned}
$$

Therefore, at $x_{0}$ we have

$$
\begin{aligned}
0 \geq & L H \\
= & \frac{1}{\tilde{u}_{\xi \xi}-v^{\prime}} L\left(\tilde{u}_{\xi \xi}-v^{\prime}\right)-\frac{1}{\left(\tilde{u}_{\xi \xi}-v^{\prime}\right)^{2}} \tilde{u}^{i j} D_{i}\left(\tilde{u}_{\xi \xi}-v^{\prime}\right) D_{j}\left(\tilde{u}_{\xi \xi}-v^{\prime}\right) \\
& +\alpha \tilde{u}^{i j} D_{i k} u D_{j k} u+\alpha D_{k} u L u_{k}+\kappa L \Phi .
\end{aligned}
$$

By a direct computation and using (17)-(19), we have

$$
\begin{aligned}
L u_{\xi \xi} \geq & \tilde{u}^{i k} \tilde{u}^{j l} D_{\xi} \tilde{u}_{i j} D_{\xi} \tilde{u}_{k l}+\tilde{u}^{i j} D_{p_{k} p_{l}} A_{i j} D_{\xi} u_{k} D_{\xi} u_{l} \\
& -C\left[\left(1+\tilde{u}_{i i}\right) \mathcal{T}+\left(\tilde{u}_{i i}\right)^{2}\right]-C\left(1+\tilde{u}_{i i}\right) B^{-\frac{1}{n-1}} \\
\geq & \tilde{u}^{i k} \tilde{u}^{j l} D_{\xi} \tilde{u}_{i j} D_{\xi} \tilde{u}_{k l}-C\left[\left(1+\tilde{u}_{i i}\right) \mathcal{T}+\left(\tilde{u}_{i i}\right)^{2}\right]-C\left(1+\tilde{u}_{i i}\right) B^{-\frac{1}{n-1}},
\end{aligned}
$$


where the first inequality is established by (21) in Lemma 2.1, and the second inequality is obtained by using the A3w condition. Note that the term $\sum_{k=1}^{n} \tilde{B}_{p_{k}} D_{i i} u_{k}$ in (21) does not appear on the right-hand side of the first inequality in (57) since it can be subtracted using the definition of the linearized operator $L$ in (23). By a direct computation, we obtain

$$
\begin{aligned}
\left|L A_{\xi \xi}\right| & \leq C\left[\left(1+\tilde{u}_{i i}\right) \mathcal{T}+\tilde{u}_{i i}\right]+\left|\frac{B_{p_{l}}}{B} A_{\xi \xi, l}\right| \\
& \leq C\left[\left(1+\tilde{u}_{i i}\right) \mathcal{T}+\tilde{u}_{i i}\right]+C\left(1+\tilde{u}_{i i}\right) B^{-\frac{1}{n-1}}
\end{aligned}
$$

where (20) in Lemma 2.1 is used in the second inequality. It follows from (16) and (20) in Lemma 2.1 that

$$
\begin{aligned}
\left|L v^{\prime}\right| & \leq C \mathcal{T}+C\left|L\left(u_{k}\right)\right| \\
& \leq C \mathcal{T}+C B^{-\frac{1}{n-1}} .
\end{aligned}
$$

Note that there is no $1+\tilde{u}_{i i}$ in the coefficient of $B^{-\frac{1}{n-1}}$ in (59) since the term $\tilde{B}_{p_{l}} D_{l} u_{k}$ is already subtracted in $L\left(u_{k}\right)$.

Hence, combining (57), (58), and (59), we have

$$
L\left(\tilde{u}_{\xi \xi}-v^{\prime}\right) \geq \tilde{u}^{i k} \tilde{u}^{j l} D_{\xi} \tilde{u}_{i j} D_{\xi} \tilde{u}_{k l}-C\left[\left(1+\tilde{u}_{i i}\right) \mathcal{T}+\tilde{u}_{i i}^{2}\right]-C\left(1+\tilde{u}_{i i}\right) B^{-\frac{1}{n-1}} .
$$

By Cauchy's inequality, we obtain

$$
\tilde{u}^{i j} D_{i}\left(\tilde{u}_{\xi \xi}-v^{\prime}\right) D_{j}\left(\tilde{u}_{\xi \xi}-v^{\prime}\right) \leq(1+\theta) \tilde{u}^{i j} D_{i} \tilde{u}_{\xi \xi} D_{j} \tilde{u}_{\xi \xi}+C(\theta) \tilde{u}^{i j} D_{i} v^{\prime} D_{j} v^{\prime}
$$

for any $\theta>0$, where $C(\theta)$ is a positive constant depending on $\theta$. Inserting (48), (60), and (61) into (56), by calculations, we get

$$
\begin{aligned}
0 \geq & L H \\
\geq & \frac{1}{\tilde{u}_{\xi \xi}-v^{\prime}} \tilde{u}^{i k} \tilde{u}^{j l} D_{\xi} \tilde{u}_{i j} D_{\xi} \tilde{u}_{k l}-\frac{1+\theta}{\left(\tilde{u}_{\xi \xi}-v^{\prime}\right)^{2}} \tilde{u}^{i j} D_{i} \tilde{u}_{\xi \xi} D_{j} \tilde{u}_{\xi \xi} \\
& -C \frac{1}{\tilde{u}_{\xi \xi}-v^{\prime}}\left\{\left[\left(1+\tilde{u}_{i i}\right) \mathcal{T}+\left(\tilde{u}_{i i}\right)^{2}\right]+C\left(1+\tilde{u}_{i i}\right) B^{-\frac{1}{n-1}}\right\} \\
& -\frac{C(\theta)}{\left(\tilde{u}_{\xi \xi}-v^{\prime}\right)^{2}} \tilde{u}^{i j} D_{i} v^{\prime} D_{j} v^{\prime}-C \alpha\left(B^{-\frac{1}{n-1}}+\mathcal{T}\right) \\
& +\alpha \sum_{i=1}^{n} \tilde{u}_{i i}+\kappa\left[\epsilon_{1} \mathcal{T}-C\left(B^{-\frac{1}{2(n-1)}}+1\right)\right] .
\end{aligned}
$$

Next, we shall deal with the terms on the right-hand side of (62). Without loss of generality, we assume that $\left\{\tilde{u}_{i j}\right\}$ is diagonal at $x_{0}$ with the maximum eigenvalue $\tilde{u}_{11}$. We can always assume that $\tilde{u}_{11}>1$ and is as large as we want; otherwise we are done. Since $v^{\prime}$ is bounded, $\tilde{u}_{11}$ and $\tilde{u}_{\xi \xi}$ are comparable in the sense that, for any $\theta>0$, there exists a further constant $C(\theta)$ such that

$$
\left|\tilde{u}_{11}-\tilde{u}_{\xi \xi}+v^{\prime}\right|<\theta \tilde{u}_{11}
$$


if $\tilde{u}_{11}>C(\theta)$. Therefore, using (63), we have

$$
\begin{aligned}
& \frac{1}{\tilde{u}_{\xi \xi}-v^{\prime}}\left[\left(1+\tilde{u}_{i i}\right) \mathcal{T}+\left(\tilde{u}_{i i}\right)^{2}\right] \\
& \leq \frac{1}{(1-\theta) \tilde{u}_{11}}\left[\left(1+\tilde{u}_{i i}\right) \mathcal{T}+\left(\tilde{u}_{i i}\right)^{2}\right] \\
& \leq C\left(\mathcal{T}+\sum_{i=1}^{n} \tilde{u}_{i i}\right)
\end{aligned}
$$

for some constant $C$ if $\theta \in(0,1 / 2)$.

We shall treat the first two terms on the right-hand side of (62). For this purpose, we will make a more detailed calculations than [15] since the equality $D_{i} A_{\xi \xi}=D_{\xi} A_{i \xi}$ does not hold in general. Because in (62) the vector $\xi$ is not equal to $e_{1}$, the situation here is different from the formula below (24) in [21]. Next, we divide the discussions into the two cases (a) and (b) assumed in the statement of this theorem.

In case $(\mathrm{a}), A_{i j}=f_{i j}\left(x, u_{i}\right) \delta_{i j}$. We define

$$
\mathcal{P}:=\frac{1}{\tilde{u}_{11}} \tilde{u}^{i k} \tilde{u}^{j l} D_{\xi} \tilde{u}_{i j} D_{\xi} \tilde{u}_{k l}-\frac{1-2 \theta}{1-\theta} \frac{1}{\tilde{u}_{11}^{2}} \tilde{u}^{i j} D_{i} \tilde{u}_{\xi \xi} D_{j} \tilde{u}_{\xi \xi},
$$

we shall get a lower bound of the quantity $\mathcal{P}$ in terms of $\mathcal{T}$. We define the matrix

$$
\left\{a_{i k}\right\}:=\left\{\tilde{u}^{j l} D_{\xi} \tilde{u}_{i j} D_{\xi} \tilde{u}_{k l}\right\}
$$

and let $\Lambda$ be its maximum eigenvalue. For the first term of $\mathcal{P}$, we have

$$
\frac{1}{\tilde{u}_{11}} \tilde{u}^{i k} \tilde{u}^{j l} D_{\xi} \tilde{u}_{i j} D_{\xi} \tilde{u}_{k l}=\frac{\operatorname{trace}\left\{\tilde{u}^{i k} a_{k j}\right\}}{\tilde{u}_{11}} \geq \frac{\tilde{u}^{11} \Lambda}{\tilde{u}_{11}}=\frac{\Lambda}{\tilde{u}_{11}^{2}} .
$$

For the second term of $\mathcal{P}$, using $\left\{\tilde{u}_{i j}\right\}:=\left\{u_{i j}-A_{i j}\right\}$, we have

$$
\begin{aligned}
\frac{1-}{1-}-\theta & \frac{1}{\tilde{u}_{11}^{2}} \tilde{u}^{i j} D_{i} \tilde{u}_{\xi \xi} D_{j} \tilde{u}_{\xi \xi} \\
= & \frac{1-2 \theta}{1-\theta} \frac{1}{\tilde{u}_{11}^{2}} \tilde{u}^{i j}\left(D_{\xi} u_{k i}-D_{i} A_{k \xi}\right)\left(D_{\xi} u_{l j}-D_{i} A_{l \xi}\right) \xi_{k} \xi_{l} \\
= & \frac{1-2 \theta}{1-\theta} \frac{1}{\tilde{u}_{11}^{2}} \tilde{u}^{i j}\left[D_{\xi}\left(u_{i k}-A_{i k}\right)+\left(D_{\xi} A_{k i}-D_{i} A_{k \xi}\right)\right] \\
& \cdot\left[D_{\xi}\left(u_{j l}-A_{j l}\right)+\left(D_{\xi} A_{j l}-D_{j} A_{l \xi}\right)\right] \xi_{k} \xi_{l} \\
= & \frac{1-2 \theta}{1-\theta} \frac{1}{\tilde{u}_{11}^{2}} \tilde{u}^{i j} D_{\xi} \tilde{u}_{i \xi} D_{\xi} \tilde{u}_{j \xi}+\frac{1-2 \theta}{1-\theta} \frac{2}{\tilde{u}_{11}^{2}} \tilde{u}^{i j} D_{\xi} \tilde{u}_{i \xi}\left(D_{\xi} A_{j \xi}-D_{j} A_{\xi \xi}\right) \\
& +\frac{1-2 \theta}{1-\theta} \frac{1}{\tilde{u}_{11}^{2}} \tilde{u}^{i j}\left(D_{\xi} A_{i \xi}-D_{i} A_{\xi \xi}\right)\left(D_{\xi} A_{j \xi}-D_{j} A_{\xi \xi}\right) \\
\leq & \frac{1}{\tilde{u}_{11}^{2}} \tilde{u}^{i j} D_{\xi} \tilde{u}_{i \xi} D_{\xi} \tilde{u}_{j \xi}+\frac{1-2 \theta}{\theta} \frac{1}{\tilde{u}_{11}^{2}} \tilde{u}^{i j}\left(D_{\xi} A_{i \xi}-D_{i} A_{\xi \xi}\right)\left(D_{\xi} A_{j \xi}-D_{j} A_{\xi \xi}\right)
\end{aligned}
$$


where the last inequality is valid by using Cauchy's inequality. We now calculate the terms $D_{i} A_{\xi \xi}$ and $D_{\xi} A_{i \xi}$. For general $A(x, D u)$, we have

$$
D_{i} A_{\xi \xi}=D_{x_{i}} A_{\xi \xi}+\left(D_{p_{k}} A_{\xi \xi}\right) u_{k i}, \quad \forall i=1, \ldots, n .
$$

For $A_{i j}=f_{i j}\left(x, u_{i}\right) \delta_{i j}$, we have

$$
D_{\xi} A_{i \xi}=\left(D_{x_{k}} f_{i i}\right) \xi_{i} \xi_{k}+\left(D_{p_{i}} f_{i i}\right) \xi_{i} u_{i \xi}, \quad \forall i=1, \ldots, n .
$$

Substituting (69) and (70) into the last term of (68), we have

$$
\begin{aligned}
& \frac{1-2 \theta}{\theta} \frac{1}{\tilde{u}_{11}^{2}} \tilde{u}^{i j}\left(D_{\xi} A_{i \xi}-D_{i} A_{\xi \xi}\right)\left(D_{\xi} A_{j \xi}-D_{j} A_{\xi \xi}\right) \\
& \quad=\frac{1-2 \theta}{\theta} \frac{1}{\tilde{u}_{11}^{2}} \tilde{u}^{i j}\left(D_{\xi} A_{i \xi} D_{\xi} A_{j \xi}-D_{\xi} A_{i \xi} D_{j} A_{\xi \xi}-D_{i} A_{\xi \xi} D_{\xi} A_{j \xi}+D_{i} A_{\xi \xi} D_{j} A_{\xi \xi}\right) \\
& \leq \frac{1-2 \theta}{\theta} \frac{C}{\tilde{u}_{11}^{2}}\left(\mathcal{T}+\tilde{u}_{11}\right) \\
& \leq C(\mathcal{T}+1)
\end{aligned}
$$

where $\tilde{u}^{i j} \tilde{u}_{j k}=\delta_{i k}$ and $\left\{\tilde{u}_{i j}\right\}:=\left\{u_{i j}-A_{i j}\right\}$ are used in the first inequality, and $\tilde{u}_{11} \geq \frac{1-2 \theta}{\theta}$ is assumed in the last inequality. Combining (65), (67), (68), and (71), we now get the lower bound of $\mathcal{P}$ in terms of $\mathcal{T}$,

$$
\mathcal{P} \geq \frac{1}{\tilde{u}_{11}^{2}}\left(\Lambda-a_{k l} \xi_{k} \xi_{l}\right)-C(\mathcal{T}+1) \geq-C(\mathcal{T}+1)
$$

where the definitions of the matrix $\left\{a_{i k}\right\}$ and its maximum eigenvalue $\Lambda$ are used successively. On the other hand, it follows from $D_{i} H=0$ in (54) that

$$
\begin{aligned}
\tilde{u}^{i j} D_{i} \tilde{u}_{\xi \xi} D_{j} \tilde{u}_{\xi \xi} & \leq 2 \tilde{u}^{i i}\left[\left|D_{i} v^{\prime}\right|^{2}+\left(\tilde{u}_{\xi \xi}-v^{\prime}\right)^{2}\left(\alpha D_{k} u D_{i k} u+\kappa D_{i} \Phi\right)^{2}\right] \\
& \leq 2 \tilde{u}^{i i}\left|D_{i} v^{\prime}\right|^{2}+C\left(\tilde{u}_{\xi \xi}-v^{\prime}\right)^{2}\left(\alpha^{2} \sum_{i=1}^{n} \tilde{u}_{i i}+\kappa^{2} \mathcal{T}\right)
\end{aligned}
$$

Combining (63), (72), and (73), we obtain

$$
\begin{aligned}
& \frac{1}{\tilde{u}_{\xi \xi}-v^{\prime}} \tilde{u}^{i k} \tilde{u}^{j l} D_{\xi} \tilde{u}_{i j} D_{\xi} \tilde{u}_{k l}-\frac{1+\theta}{\left(\tilde{u}_{\xi \xi}-v^{\prime}\right)^{2}} \tilde{u}^{i j} D_{i} \tilde{u}_{\xi \xi} D_{j} \tilde{u}_{\xi \xi} \\
& \geq \frac{1}{1-\theta}\left(\frac{1}{\tilde{u}_{11}} \tilde{u}^{i k} \tilde{u}^{j l} D_{\xi} \tilde{u}_{i j} D_{\xi} \tilde{u}_{k l}-\frac{1+\theta}{(1-\theta) \tilde{u}_{11}^{2}} \tilde{u}^{i j} D_{i} \tilde{u}_{\xi \xi} D_{j} \tilde{u}_{\xi \xi}\right) \\
& \geq \frac{1}{1-\theta}\left(\mathcal{P}-\frac{3 \theta}{(1-\theta) \tilde{u}_{11}^{2}} \tilde{u}^{i j} D_{i} \tilde{u}_{\xi \xi} D_{j} \tilde{u}_{\xi \xi}\right) \\
& \geq-\frac{C(\mathcal{T}+1)}{1-\theta}-\frac{3 \theta}{\left[(1-\theta) \tilde{u}_{11}\right]^{2}}\left[2 \tilde{u}^{i i}\left|D_{i} v^{\prime}\right|^{2}+C\left(\tilde{u}_{\xi \xi}-v^{\prime}\right)^{2}\left(\alpha^{2} \sum_{i=1}^{n} \tilde{u}_{i i}+\kappa^{2} \mathcal{T}\right)\right] \\
& \geq-C(\mathcal{T}+1)-C \theta \alpha^{2} \sum_{i=1}^{n} \tilde{u}_{i i}-C \theta \kappa^{2} \mathcal{T}
\end{aligned}
$$


for $\theta \in(0,1 / 2)$, where $C$ becomes a further constant in the last inequality. We can assume $\tilde{u}_{11} \geq 1$, otherwise we have already obtained the desired estimate. Similar to (37), we also have

$$
B^{-\frac{1}{n-1}} \leq \frac{\mathcal{T}}{n-1} .
$$

For the last term in (62), using Cauchy's inequality, we have

$$
\begin{array}{rl}
\epsilon_{1} & \mathcal{T}-C\left(B^{-\frac{1}{2(n-1)}}+1\right) \\
& \geq \epsilon_{1} \mathcal{T}-\epsilon B^{-\frac{1}{n-1}}-C(\epsilon) \\
& \geq \frac{\epsilon_{1}}{2} \mathcal{T}-C\left(\epsilon_{1}\right),
\end{array}
$$

where we take $\epsilon=\frac{n-1}{2} \epsilon_{1}$ and use (75) in the second inequality.

Inserting (63), (64), (74), (75), and (76) into (62), we obtain, for $\tilde{u}_{11} \geq \max \{C(\theta), 1\}$,

$$
\begin{aligned}
& \alpha \sum_{i=1}^{n} \tilde{u}_{i i}+\frac{\kappa \epsilon_{1}}{2} \mathcal{T} \\
& \quad \leq C\left\{\left[1+\kappa C\left(\epsilon_{1}\right)\right]+\left(1+\alpha^{2} \theta\right) \sum_{i=1}^{n} \tilde{u}_{i i}+\left(1+\alpha+\kappa^{2} \theta\right) \mathcal{T}\right\} .
\end{aligned}
$$

By choosing $\kappa \gg \alpha \gg 1$ and fixing a small positive $\theta=1 / \kappa^{2}$, we can get from (77) that

$$
\sum_{i=1}^{n} \tilde{u}_{i i}\left(x_{0}\right) \leq C
$$

which implies a corresponding estimate for $\left|D^{2} u\left(x_{0}\right)\right|$ in case (a).

In case (b), $\left|D_{p_{k}} A_{i j}\right|<\delta$ for all $i, j, k=1, \ldots, n$, and sufficiently small $\delta$. We define

$$
\mathcal{P}^{\prime}:=\frac{1}{\tilde{u}_{11}} \tilde{u}^{i k} \tilde{u}^{j l} D_{\xi} \tilde{u}_{i j} D_{\xi} \tilde{u}_{k l}-\frac{1}{\tilde{u}_{11}^{2}} \tilde{u}^{i j} D_{i} \tilde{u}_{\xi \xi} D_{j} \tilde{u}_{\xi \xi} .
$$

Similarly to (68), we can get

$$
\begin{aligned}
& \frac{1}{\tilde{u}_{11}^{2}} \tilde{u}^{i j} D_{i} \tilde{u}_{\xi \xi} D_{j} \tilde{u}_{\xi \xi} \\
& =\frac{1}{\tilde{u}_{11}^{2}} \tilde{u}^{i j} D_{\xi} \tilde{u}_{i \xi} D_{\xi} \tilde{u}_{j \xi}+\frac{2}{\tilde{u}_{11}^{2}} \tilde{u}^{i j} D_{\xi} \tilde{u}_{i \xi}\left(D_{\xi} A_{j \xi}-D_{j} A_{\xi \xi}\right) \\
& \quad+\frac{1}{\tilde{u}_{11}^{2}} \tilde{u}^{i j}\left(D_{\xi} A_{i \xi}-D_{i} A_{\xi \xi}\right)\left(D_{\xi} A_{j \xi}-D_{j} A_{\xi \xi}\right) .
\end{aligned}
$$

For the middle term on the right-hand side of (80), rather than using Cauchy's inequality, it can be dealt with by using $D_{\xi} \tilde{u}_{i \xi}=D_{i} u_{\xi \xi}-D_{\xi} A_{i \xi}$, (69), (70), (54), and $\left|D_{p_{k}} A_{i j}\right|<\delta$, namely

$$
\begin{aligned}
& \frac{2}{\tilde{u}_{11}^{2}} \tilde{u}^{i j} D_{\xi} \tilde{u}_{i \xi}\left(D_{\xi} A_{j \xi}-D_{j} A_{\xi \xi}\right) \\
& \quad=\frac{2}{\tilde{u}_{11}^{2}} \tilde{u}^{i j}\left[D_{i} \tilde{u}_{\xi \xi}-\left(D_{\xi} A_{i \xi}-D_{i} A_{\xi \xi}\right)\right]\left(D_{\xi} A_{j \xi}-D_{j} A_{\xi \xi}\right)
\end{aligned}
$$




$$
\begin{aligned}
& \leq \frac{2}{\tilde{u}_{11}^{2}} \tilde{u}^{i j} D_{i} \tilde{u}_{\xi \xi}\left(D_{\xi} A_{j \xi}-D_{j} A_{\xi \xi}\right) \\
& =\frac{2}{\tilde{u}_{11}^{2}} \tilde{u}^{i j}\left[D_{i} v^{\prime}-\left(\tilde{u}_{\xi \xi}-v^{\prime}\right)\left(\alpha D_{k} u D_{i k} u+\kappa D_{i} \Phi\right)\right]\left(D_{\xi} A_{j \xi}-D_{j} A_{\xi \xi}\right) \\
& \leq C[1+\delta(\alpha+\kappa \mathcal{T})]
\end{aligned}
$$

provided $\tilde{u}_{11} \geq 1$. We can analyze the last term on the right-hand side of (80) for general matrix $A$ to get

$$
\frac{1}{\tilde{u}_{11}^{2}} \tilde{u}^{i j}\left(D_{\xi} A_{i \xi}-D_{i} A_{\xi \xi}\right)\left(D_{\xi} A_{j \xi}-D_{j} A_{\xi \xi}\right) \leq C(\mathcal{T}+1)
$$

provided $\tilde{u}_{11} \geq 1$. Combining (79), (80), (81), and (82), we obtain

$$
\begin{aligned}
\mathcal{P}^{\prime} & \geq \frac{1}{\tilde{u}_{11}^{2}}\left(\Lambda-a_{k l} \xi_{k} \xi_{l}\right)-C[1+\mathcal{T}+\delta(\alpha+\kappa \mathcal{T})] \\
& \geq-C[1+\mathcal{T}+\delta(\alpha+\kappa \mathcal{T})] .
\end{aligned}
$$

Combining (63), (83), and (73), we obtain

$$
\begin{aligned}
& \frac{1}{\tilde{\tilde{u}}_{\xi \xi}-v^{\prime}} \tilde{u}^{i k} \tilde{u}^{j l} D_{\xi} \tilde{u}_{i j} D_{\xi} \tilde{u}_{k l}-\frac{1+\theta}{\left(\tilde{u}_{\xi \xi}-v^{\prime}\right)^{2}} \tilde{u}^{i j} D_{i} \tilde{u}_{\xi \xi} D_{j} \tilde{u}_{\xi \xi} \\
& \geq \frac{1}{1-\theta}\left(\frac{1}{\tilde{u}_{11}} \tilde{u}^{i k} \tilde{u}^{j l} D_{\xi} \tilde{u}_{i j} D_{\xi} \tilde{u}_{k l}-\frac{1+\theta}{(1-\theta) \tilde{u}_{11}^{2}} \tilde{u}^{i j} D_{i} \tilde{u}_{\xi \xi} D_{j} \tilde{u}_{\xi \xi}\right) \\
& \geq \frac{1}{1-\theta}\left(\mathcal{P}^{\prime}-\frac{2 \theta}{(1-\theta) \tilde{u}_{11}^{2}} \tilde{u}^{i j} D_{i} \tilde{u}_{\xi \xi} D_{j} \tilde{u}_{\xi \xi}\right) \\
& \geq-C[1+\mathcal{T}+\delta(\alpha+\kappa \mathcal{T})]-C \theta \alpha^{2} \sum_{i=1}^{n} \tilde{u}_{i i}-C \theta \kappa^{2} \mathcal{T}
\end{aligned}
$$

for $\theta \in(0,1 / 2)$. Using the estimate in (83) and deducing as in case (a), for $\tilde{u}_{11} \geq$ $\max \{C(\theta), 1\}$, we obtain in place of $(77)$

$$
\begin{aligned}
& \alpha \sum_{i=1}^{n} \tilde{u}_{i i}+\frac{\kappa \epsilon_{1}}{2} \mathcal{T} \\
& \quad \leq C\left\{\left[1+\kappa C\left(\epsilon_{1}\right)+\delta \alpha\right]+\left(1+\alpha^{2} \theta\right) \sum_{i=1}^{n} \tilde{u}_{i i}+\left(1+\alpha+\kappa^{2} \theta+\kappa \delta\right) \mathcal{T}\right\} .
\end{aligned}
$$

By choosing $\alpha=2 C+1$ and $\kappa=\frac{2[C(\alpha+3)+1]}{\epsilon_{1}}$ successively and fixing the positive constants $\theta=$ $1 / \kappa^{2}$ and $\delta=1 / \kappa$, we can get from (85) that (78) holds again, which implies a corresponding estimate for $\left|D^{2} u\left(x_{0}\right)\right|$ in case (b).

Next, we consider the case $x_{0} \in \partial \Omega$, namely the function $G$ in (50) attains its maximum over $\bar{\Omega}$ at $x_{0} \in \partial \Omega$ and a unit vector $\xi$. The estimation of the rest of the Hessian $D^{2} u$ splits into two stages according to a different direction of $\xi$. 
Case (i). $\xi$ tangential. Since $v^{\prime}\left(x_{0}, \xi\right)=0$, at $x_{0}$ we obtain

$$
\begin{aligned}
0 \geq & D_{v} G \\
= & D_{v}\left\{\left[\tilde{u}_{\xi \xi}-v^{\prime}(x, \xi)\right] e^{\frac{\alpha}{2}|D u|^{2}+\kappa \Phi}\right\} \\
= & e^{\frac{\alpha}{2}|D u|^{2}+\kappa \Phi}\left\{\left(\tilde{u}_{\xi \xi}-v^{\prime}(x, \xi)\right) D_{v}\left(\frac{\alpha}{2}|D u|^{2}+\kappa \Phi\right)+D_{v}\left(\tilde{u}_{\xi \xi}-v^{\prime}(x, \xi)\right)\right\} \\
= & e^{\frac{\alpha}{2}|D u|^{2}+\kappa \Phi}\left\{\left[\alpha D_{k} u D_{\nu}\left(D_{k} u\right)+\kappa D_{\nu} \Phi\right] \tilde{u}_{\xi \xi}+D_{\nu} u_{\xi \xi}-D_{v}\left(A_{\xi \xi}+v^{\prime}\right)\right\} \\
= & e^{\frac{\alpha}{2}|D u|^{2}+\kappa \Phi}\left\{\left[\kappa D_{\nu} \Phi+\alpha D_{k} u\left(\varphi_{k}-D_{i} u D_{k} v_{i}\right)\right] \tilde{u}_{\xi \xi}\right. \\
& \left.+D_{\nu} u_{\xi \xi}-D_{v}\left(A_{\xi \xi}+v^{\prime}\right)\right\} \\
\geq & e^{\frac{\alpha}{2}|D u|^{2}+\kappa \Phi}\left\{\left(\kappa c_{0}-\alpha M\right) \tilde{u}_{\xi \xi}+D_{\nu} u_{\xi \xi}-D_{v}\left(A_{\xi \xi}+v^{\prime}\right)\right\}
\end{aligned}
$$

where $c_{0}=K a \inf \left(e^{K(\underline{u}-u)}\right), M=\max _{x \in \partial \Omega}\left|D_{k} u\left(\varphi_{k}-D_{i} u D_{k} v_{i}\right)\right|$. The above inequality gives a relationship between $\tilde{u}_{\xi \xi}\left(x_{0}\right)$ and $D_{\nu} u_{\xi \xi}\left(x_{0}\right)$ at $x_{0}$, namely

$$
D_{\nu} u_{\xi \xi} \leq-\left(\kappa c_{0}-\alpha M\right) \tilde{u}_{\xi \xi}+D_{\nu}\left(A_{\xi \xi}+v^{\prime}\right)
$$

On the other hand, by tangential differentiating the boundary condition twice, we obtain

$$
\left(D_{k} u\right) \delta_{i} \delta_{j} v_{k}+\left(\delta_{i} D_{k} u\right) \delta_{j} v_{k}+\left(\delta_{j} D_{k} u\right) \delta_{i} v_{k}+v_{k} \delta_{i} \delta_{j} D_{k} u=\delta_{i} \delta_{j} \varphi \quad \text { on } \partial \Omega
$$

Hence, for the tangential direction $\xi$ at $x_{0}$, we have

$$
\begin{aligned}
D_{\nu} u_{\xi \xi} & \geq-2\left(\delta_{i} v_{k}\right) D_{j k} u \xi_{i} \xi_{j}+\left(\delta_{i} v_{j}\right) \xi_{i} \xi_{j} D_{\nu v} u-C \\
& \geq-2\left(\delta_{i} v_{k}\right) D_{j k} u \xi_{i} \xi_{j}-C
\end{aligned}
$$

where the double normal derivative estimate (46) on $\partial \Omega$ is used in the second inequality. Thus, at $x_{0}$ we have

$$
\begin{aligned}
\left(\kappa c_{0}-\alpha M\right) \tilde{u}_{\xi \xi} & \leq 2\left(\delta_{i} v_{k}\right) D_{j k} u \xi_{i} \xi_{j}+D_{\nu}\left(A_{\xi \xi}+v^{\prime}\right)+C \\
& \leq 2\left(\delta_{i} v_{k}\right) D_{j k} u \xi_{i} \xi_{j}+C\left|D D_{\nu} u\right|+C \\
& \leq 2\left(\delta_{i} v_{k}\right) D_{j k} u \xi_{i} \xi_{j}+C,
\end{aligned}
$$

where the mixed tangential normal derivative estimate (28) and the double normal derivative estimate (46) are used to obtain the last inequality, and the constant $C$ changes from line to line. Without loss of generality, we can assume the normal at $x_{0}$ to be $v=(0, \ldots, 1)$, and we can assume $\left\{\tilde{u}_{i j}\left(x_{0}\right)\right\}_{i, j<n}$ is diagonal with maximum eigenvalue $\tilde{u}_{11}\left(x_{0}\right)>1$. Then, at $x_{0}$ we obtain

$$
\left(\kappa c_{0}-\alpha M\right) \tilde{u}_{\xi \xi} \leq C\left(\tilde{u}_{11}+1\right) .
$$

Since $G\left(x_{0}, e_{1}\right) \leq G\left(x_{0}, \xi\right)$, we have

$$
\tilde{u}_{11}\left(x_{0}\right) \leq \tilde{u}_{\xi \xi}\left(x_{0}\right)+v^{\prime}\left(x_{0}, e_{1}\right)-v^{\prime}\left(x_{0}, \xi\right) .
$$


Combining (91) and (92) and choosing $\kappa \geq \frac{2}{c_{0}}(\alpha M+C)$, we obtain

$$
u_{\xi \xi}\left(x_{0}\right) \leq C
$$

Case (ii). $\xi$ non-tangential. We write $\xi$ as

$$
\xi=\alpha \tau+\beta \nu,
$$

where $\alpha=\xi \cdot \tau,|\tau|=1, \tau \cdot v=0, \beta=\xi \cdot v \neq 0$, and

$$
\alpha^{2}+\beta^{2}=1
$$

Therefore, at $x_{0}$ we have

$$
\begin{aligned}
D_{\xi \xi} \tilde{u} & =\alpha^{2} \tilde{u}_{\tau \tau}+\beta^{2} \tilde{u}_{v v}+2 \alpha \beta \tilde{u}_{\tau v} \\
& =\alpha^{2} \tilde{u}_{\tau \tau}+\beta^{2} \tilde{u}_{v v}+v^{\prime}(x, \xi),
\end{aligned}
$$

where the definition of $v^{\prime}$ in (51) is used. Since $G\left(x_{0}, \tau\right) \leq G\left(x_{0}, \xi\right)$, we obtain

$$
\begin{aligned}
G\left(x_{0}, \xi\right) & =\alpha^{2} G\left(x_{0}, \tau\right)+\beta^{2} G\left(x_{0}, v\right) \\
& \leq \alpha^{2} G\left(x_{0}, \xi\right)+\beta^{2} G\left(x_{0}, \nu\right) .
\end{aligned}
$$

Using (95) in (97), we get

$$
G\left(x_{0}, \xi\right) \leq G\left(x_{0}, v\right)
$$

which implies

$$
D_{\xi \xi} u\left(x_{0}\right) \leq C+D_{\nu \nu} u\left(x_{0}\right) \leq C,
$$

where the double normal derivative estimate (46) on $\partial \Omega$ is used again.

Therefore, we can conclude from the two cases (i) and (ii) that if $G$ attains its global maximum at $x_{0} \in \partial \Omega$ and a unit vector $\xi$, then $D_{\xi \xi} u\left(x_{0}\right)$ is bounded from above. Taking (78) into account, we can derive estimate (11). Hence, Theorem 1.1 is proved.

\section{$4 C^{0}, C^{1}$ estimates and the existence}

In this section, we derive the lower order a priori derivative estimates (i.e., the $C^{0}$ and $C^{1}$ estimates) for Monge-Ampère type equations (1) with the Neumann boundary value condition (2). Using these estimates together with the second derivative estimate in Theorem 1.1, we give the proof of Theorem 1.2.

Since $B_{z}>0$, the comparison principle for the classical solutions is valid. Under the assumptions that $\underline{u}$ satisfies (4)-(5) and $\bar{u}$ satisfies (1) and $D_{\nu} \bar{u} \leq \varphi(x)$ on $\partial \Omega$, by comparison principle, we have

$$
\underline{u} \leq u \leq \bar{u}
$$

in $\bar{\Omega}$, which gives the $C^{0}$ estimate of the solution $u$. 
The $C^{1}$ estimate is established in $[15,22]$ in a general form. For convenience, we repeat it here.

Theorem 4.1 Let $u \in C^{2}(\Omega) \cap C^{1,1}(\bar{\Omega})$ satisfy

$$
D^{2} u \geq-\mu_{0}\left(1+|D u|^{2}\right) I
$$

in a $C^{2}$ domain $\Omega \subset \mathbb{R}^{n}$, with $D_{\nu} u \geq-\delta$ on $\partial \Omega$, where $\mu_{0}$ and $\delta$ are nonnegative constants. Then we have the estimate

$$
|D u| \leq C
$$

where $C$ depends on $\mu_{0}, \delta, \Omega$, and $\sup |u|$.

From the ellipticity and the quadratic lower bound for $A$ in (13), it is easy to get inequality (101). Setting $\delta=\max \left\{-\min _{\partial \Omega} \varphi, 0\right\}, u$ satisfying (2) automatically satisfies $D_{v} u \geq-\delta$ on $\partial \Omega$. Hence, the gradient estimate (102) holds for the solution $u$ of problem (1)-(2).

With the preparations of a priori estimates (100), (102), and (11), we are able to prove the existence result in Theorem 1.2.

Proof of Theorem 1.2 We shall prove the existence of a viscosity solution $u$ of the Neumann problem of the degenerate Monge-Ampère equation (1)-(2) by approximations.

We consider the following approximating problem:

$$
\begin{aligned}
& \operatorname{det}\left[D^{2} u-A(\cdot, D u)\right]=B_{\epsilon}>0, \quad \text { in } \Omega, \\
& D_{\nu} u=\varphi(x), \quad \text { on } \partial \Omega,
\end{aligned}
$$

where

$$
B_{\epsilon}(\cdot, u, D u):=B(\cdot, u, D u)+\epsilon
$$

for small positive constant $\epsilon$. If $B$ satisfies conditions (6)-(8), it is obvious that $B_{\epsilon}$ still satisfies the same conditions.

By Remark 1, there exists a unique elliptic solution $u_{\epsilon} \in C^{3, \alpha}$ of problem (103), where $\alpha \in(0,1)$. Since $\bar{u}$ is still the supersolution of problem (103), and for sufficiently small $\epsilon>0, \underline{u}$ is still the subsolution of problem (103) as well, we get the uniform estimate

$$
\underline{u} \leq u_{\epsilon} \leq \bar{u}
$$

for sufficiently small $\epsilon>0$. Notice that the gradient estimate in Theorem 4.1 is independent of the lower bound of the right-hand side term of the equation. Therefore, we have

$$
\left|D u_{\epsilon}\right| \leq C
$$

where the constant $C$ is independent of $\epsilon$. By Theorem 1.1, we obtain the uniform second derivative estimate

$$
\sup _{\Omega}\left|D^{2} u_{\epsilon}\right| \leq C
$$


From the remarks in the paragraph below Theorem 1.1 (or from the proof of Theorem 1.1), we know that the constant $C$ in (106) is independent of $\epsilon$. With the uniform estimates (104), (105), and (106), we can pass the limit to $u_{\epsilon}$. Now letting $\epsilon \rightarrow 0$ (passing to a subsequence if necessary), we get a viscosity solution $u$ of the degenerate Monge-Ampère equation (1) with the Neumann boundary condition (2), which is degenerate elliptic and satisfies the estimate

$$
\|u\|_{C^{1,1}(\bar{\Omega})} \leq C .
$$

Therefore, the proof of Theorem (1.2) is completed.

Remark 7 In Theorem 1.2, we proved the existence of a viscosity solution $u \in C^{1,1}(\bar{\Omega})$ by smooth approximations. Such uniqueness of viscosity solutions in our $A_{z}=0, B_{z}>0$, and $\varphi_{z}=0$ case is not covered in Sect. 4 of [23]. It would be interesting to investigate the uniqueness of the viscosity solutions of the degenerate Monge-Ampère equation (1) satisfying the Neumann boundary condition (2).

Remark 8 In the current paper, we show that "the existence of a subsolution implies the existence of a solution", which is the new point of the existence result to the Neumann problem (1)-(2) of degenerate Monge-Ampère type equations. This idea is also new even for nondegenerate Monge-Ampère type equations. For the nondegenerate case in [15], for a more general problem with $A$ and $\varphi$ depending also on $u$,

$$
\begin{aligned}
& \operatorname{det}\left[D^{2} u-A(\cdot, u, D u)\right]=B(\cdot, u, D u) \quad \text { in } \Omega, \\
& D_{\nu} u=\varphi(\cdot, u) \quad \text { on } \partial \Omega,
\end{aligned}
$$

the result is that "the existence of a supersolution implies the existence of a solution". If we consider such a general problem (108) in the degenerate elliptic setting, the supersolution $u$ is only degenerate elliptic and is not enough to construct a barrier function. It needs new ideas to solve the general Neumann problem (108) in the degenerate case.

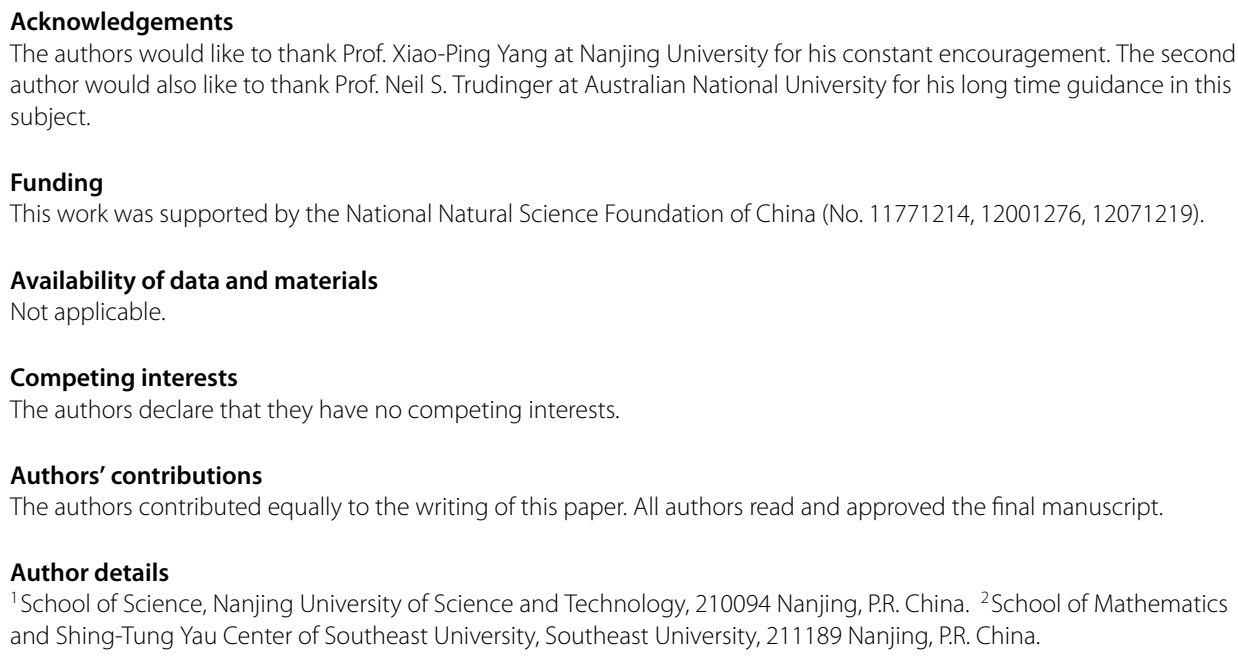




\section{Publisher's Note}

Springer Nature remains neutral with regard to jurisdictional claims in published maps and institutional affiliations.

Received: 16 September 2020 Accepted: 7 January 2021 Published online: 21 January 2021

\section{References}

1. Ma, X.N., Trudinger, N.S., Wang, X.-J.: Regularity of potential functions of the optimal transportation problem. Arch. Ration. Mech. Anal. 177, 151-183 (2005)

2. Loeper, G.: On the regularity of solutions of optimal transportation problems. Acta Math. 202, 241-283 (2009)

3. Andriyanova, E.: On the Dirichlet problem for degenerate elliptic Monge-Ampère type equations. Calc. Var. Partial Differ. Equ. 58, 181 (2019)

4. Bao, J.G.: The Dirichlet problem for the degenerate elliptic Monge-Ampère equation. J. Math. Anal. Appl. 238, 168-178 (1999)

5. Błocki, Z: Interior regularity of the degenerate Monge-Ampère equations. Bull. Aust. Math. Soc. 68, 81-92 (2003)

6. Guan, P.F.: $C^{2}$ a priori estimates for degenerate Monge-Ampère equations. Duke Math. J. 86, 323-346 (1997)

7. Guan, P.F., Trudinger, N.S., Wang, X.-.:: On the Dirichlet problem for degenerate Monge-Ampère equations. Acta Math. 182, 87-104 (1999)

8. Shi, J.H., Jiang, F.D.: Pogorelov estimates for the Monge-Ampère equations. Proc. Am. Math. Soc. 147, 2561-2571 (2019)

9. Jiang, F.D., Trudinger, N.S.: On Pogorelov estimates in optimal transportation and geometric optics. Bull. Math. Sci. 4 407-431 (2014)

10. Jiang, F.D., Trudinger, N.S.: On the second boundary value problem for Monge-Ampère type equations and geometric optics. Arch. Ration. Mech. Anal. 229, 547-567 (2018)

11. Trudinger, N.S.: Recent developments in elliptic partial differential equations of Monge-Ampère type. In: Proceedings of the International Congress of Mathematicians, vol. 3, pp. 291-302. ICM, Madrid (2006)

12. Huang, Y., Jiang, F.D., Liu, J.K.: Boundary C2, Estimates for Monge-Ampère Type Equations. Birkhäuser, Boston (2001)

13. Jiang, F.D., Trudinger, N.S., Yang, X.P.: On the Dirichlet problem for Monge-Ampère type equations. Calc. Var. Partial Differ. Equ. 49, 1223-1236 (2014)

14. Lions, P.L., Trudinger, N.S., Urbas, J.I.E.: The Neumann problem for equations of Monge-Ampère type. Commun. Pure Appl. Math. 39, 539-563 (1986)

15. Jiang, F.D., Trudinger, N.S., Xiang, N.: On the Neumann problem for Monge-Ampère type equations. Can. J. Math. 68 , 1334-1361 (2016)

16. Trudinger, N.S., Urbas, J.: On the second derivative estimates for equations of Monge-Ampère type. Bull. Aust. Math Soc. 30, 321-334 (1984)

17. Caffarelli, L.A., Nirenberg, L., Spruck, J.: The Dirichlet problem for the degenerate Monge-Ampère equation. Rev. Mat. Iberoam. 2, 19-27 (1986)

18. Li, Q.-R., Wang, X.-J.: Regularity of the homogeneous Monge-Ampère equation. Discrete Contin. Dyn. Syst. 35 6069-6084 (2015)

19. Jiang, F.D., Shi, J.H., Yang, X.-P.: Interior $C^{1,1}$ regularity of solutions to a class of doubly degenerate Monge-Ampère type equations. Preprint (2020)

20. Jiang, F.D., Trudinger, N.S.: Oblique boundary value problems for augmented Hessian equations II. Nonlinear Anal. $154,148-173(2017)$

21. Liu, J.K., Trudinger, N.S.: On Pogorelov estimates for Monge-Ampère type equations. Discrete Contin. Dyn. Syst. 28, 1121-1135 (2010)

22. Jiang, F.D., Xiang, N., Xu, J..: Gradient estimates for Neumann boundary value problem of Monge-Ampère type equations. Commun. Contemp. Math. 19, 1650041 (2017)

23. Jiang, F.D., Trudinger, N.S.: Oblique boundary value problems for augmented Hessian equations III. Commun. Partial Differ. Equ. 44, 708-748 (2019)

\section{Submit your manuscript to a SpringerOpen ${ }^{\circ}$ journal and benefit from:}

- Convenient online submission

- Rigorous peer review

- Open access: articles freely available online

- High visibility within the field

- Retaining the copyright to your article

Submit your next manuscript at $\boldsymbol{~ s p r i n g e r o p e n . c o m ~}$ 